\title{
Which neuropsychiatric and behavioural features distinguish frontal and temporal variants of frontotemporal dementia from Alzheimer's disease?
}

Sasha Bozeat, Carol A Gregory, Matthew A Lambon Ralph, John R Hodges

\begin{abstract}
Objectives-To investigate the prevalence of changes in mood, personality, and behaviour in frontotemporal dementia (FTD) and Alzheimer's disease (AD) and hence, which features reliably distinguish between them. To establish whether the frontal and temporal variants of FTD are characterised by different behavioural changes.
\end{abstract}

Methods-A questionnaire was designed to assess a wide range of neuropsychiatric changes; it incorporated features reported in previous studies of FTD and components of the neuropsychiatric inventory. ${ }^{1}$ This was completed by 37 carers of patients with Alzheimer's disease (AD) and 33 patients with frontotemporal dementia (FTD), comprising 20 with temporal variant FTD (tv FTD) or semantic dementia and 13 with frontal variant FTD (fv FTD). An exploratory principal components factor analysis and discriminant function analysis was applied.

Results-Factor analysis showed four robust and meaningful symptom clusters: factor 1-stereotypic and eating behaviour; factor 2-executive dysfunction and self care; factor 3-mood changes; factor 4-loss of social awareness. Only stereotypic and altered eating behaviour and loss of social awareness reliably differentiated AD from FTD with no effect of disease severity. By contrast, executive dysfunction, poor self care, and restlessness showed a significant effect of disease severity only, with the more impaired patients scoring more highly. Changes in mood were found to be equally prevalent in the three patient groups. Analysis of individual symptoms showed increased rates of mental rigidity and depression in the patients with semantic dementia compared with those with fv FTD. Conversely, the latter group showed greater disinhibition. Discriminant function analysis correctly classified $71.4 \%$ overall and $86.5 \%$ of the patients with AD.

Conclusions-This questionnaire disclosed striking differences between patients with FTD and AD, but only stereotypic behaviour, changes in eating preference, disinhibition, and features of poor social awareness reliably separated the groups. The patients with fv FTD and semantic dementia were behaviourally very similar, reflecting the involvement of a common network, the ventral frontal lobe, temporal pole, and amygdala. Dysexecutive symptoms and poor self care were found to be affected by the severity of the disease, reflecting perhaps spread to dorsolateral prefrontal areas relatively late in the course of both FTD and AD. This questionnaire may be of value in the diagnosis and the monitoring of therapies. (F Neurol Neurosurg Psychiatry 2000;69:178-186)

Keywords: frontotemporal dementia; Alzheimer's disease; semantic dementia; neuropsychiatry

Frontotemporal dementia (FTD) is the term currently used in preference to Pick's disease to describe a range of non-Alzheimer dementias producing focal lobar atrophy involving the frontal lobe and/or the temporal lobes. ${ }^{2-4}$ Frontotemporal dementia is the second commonest form of primary dementia in the presenium and has an enormous impact on carers as it produces marked changes in personality, behaviour, and communication abilities. ${ }^{5}$ Yet, compared to the vast literature on Alzheimer's disease (AD), there have been relatively few systematic studies of FTD and even fewer have compared substantial groups of patients with FTD and AD.

Although often considered a unitary syndrome, numerous studies have characterised the two major presentations of FTD which reflect the predominant locus of pathology: the progressive change in personality coupled with executive dysfunction associated with the frontal variant of FTD ( $\mathrm{fv}$ FTD) and the progressive fluent aphasia with breakdown in semantic knowledge (semantic dementia) found in patients with the temporal lobe variant of FTD. ${ }^{56}$ Cases with both variants were clearly described by Arnold Pick over 100 years ago, ${ }^{7-9}$ but their delineation and characterisation has occurred only in the past decade or so. In the 1980s workers in Lund and Manchester simultaneously recognised that a significant proportion of patients with dementia presented with a frontal syndrome and coined the term dementia of the frontal type. This was preferred to Pick's disease as only a minority had specific intraneuronal inclusions (Pick bodies). ${ }^{10}{ }^{11}$ Other terms used to describe identical cases include frontal lobe degeneration, ${ }^{12}$ frontal lobe dementia, ${ }^{13}$ and most recently frontal variant FTD. ${ }^{14-16}$ 
Mesulam ${ }^{17}$ used the term primary progressive aphasia to refer to patients with a predominant language impairment resulting from focal atrophy. More than 100 patients have now been described with progressive aphasia and although the language disorder is heterogeneous, two fairly distinct syndromes have emerged: progressive non-fluent and progressive fluent aphasia. ${ }^{18}$ The latter is also known as semantic dementia as the primary deficit is a profound loss of conceptual knowledge underlying their anomia and progressive loss of verbal and non-verbal comprehension. Patients with semantic dementia have striking focal temporal lobe atrophy ${ }^{19}$ and the range of pathological findings are identical to that found in patients with fv FTD. ${ }^{14}{ }^{15}$ The literature on semantic dementia has focused predominantly on the cognitive and linguistic features, but a recent study suggested that changes in behaviour and personality are also common. ${ }^{20}$

The realisation that patients with identical pathology may present with either frontal or temporal lobe dysfunction, and that both features tend to emerge with time, were factors that led the Lund/Manchester groups to propose the use of the term FTD. ${ }^{2}{ }^{11}$ After the discovery of tau gene mutations in some familial cases, the use of the term FTD has increased..$^{21}$ It is, however, a blanket term which has led to it being treated as a unitary phenomenon rather than recognising the heterogeneity of its presentation. Researchers have tended to amalgamate patients, a process that is likely to blur important distinctions. ${ }^{46223}$ We think that the cognitive deficits are fundamentally different in patients with frontal pathology and those with predominantly temporal pathology $^{1624}$ and consider here the issue of whether the behavioural profiles are distinguishable.

A wide range of behavioural abnormalities have been reported in FTD including loss of insight, disinhibition, impulsivity, apathy, reduced empathy for others, poor self care, mood changes, stereotypic behaviour, mental rigidity, and changes in eating patterns. ${ }^{2325}$ Although there are many good case descriptions of patients with these behavioural changes ${ }^{11} 2627$ the prevalence and, in particular, their specificity to FTD has only just begun to be systematically explored. For instance, Levy et al used a standardised carer interview, the neuropsychiatric inventory (NPI), in an attempt to differentiate FTD and AD. ${ }^{22}$ They found that scores for disinhibition, apathy, aberrant motor behaviour, and euphoria were significantly higher in the patients with FTD. This instrument was not, however, specifically designed for FTD and does not include measures of stereotypic or ritualistic behaviour, which other workers have found to be very common in FTD (for a review see Ames et $a l^{77}$ ).In addition, the authors failed to subdivide the patients with FTD into those with the frontal and temporal variants.

Similar findings are reported by Kertesz et $a l^{28}$ based on their frontal behavioural inventory (FBI) but again, this scale does not include measures of stereotypic and ritualistic behaviour. In an analysis of the Lund/Manchester criteria, Miller et $a l^{4}$ found that stereotyped and perseverative behaviour were among the five features that best discriminated AD from FTD, but they did not distinguish between temporal and frontal variants and based their findings on a retrospective case note review.

This present study uses a newly created carer questionnaire to examine the neuropsychiatric features in FTD and AD. The two main aims were: (1) to investigate the range and frequency of behavioural changes in FTD and AD and hence, which features reliably distinguish between them; (2) to establish whether the frontal and temporal variants of FTD are characterised by different behavioural changes. Although it is clear that semantic dementia results in profound changes in language and knowledge which do not occur in those with $\mathrm{fv}$ FTD,${ }^{24} 290$ it is unclear whether the two groups differ in the range or frequency of the neuropsychiatric symptoms.

\section{Methods}

PATIENTS

Patients were identified through the Memory and Cognitive Disorders Clinic at Addenbrooke's Hospital, Cambridge, England, where they were seen by a senior neurologist (JRH), a senior psychiatrist, and a clinical neuropsychologist. In addition to a clinical assessment, all patients were given standard psychiatric rating scales, to exclude major functional psychiatric disorders such as depression and schizophrenia. The standard neuropsychological battery applied to all cases included tests of episodic memory (the Warrington recognition memory test, ${ }^{31}$ story recall from the WMS-R, ${ }^{32}$ and recall of the Rey complex figure ${ }^{33}$ ); semantic memory (category fluency and naming of 64 items chosen from the Snodgrass and Vanderwart corpus, ${ }^{34}{ }^{35}$ and The pyramids and palm trees test ${ }^{36}$ ); visuospatial and perceptual assessments (the visual object and space perception battery ${ }^{37}$ ); executive function (the modified Wisconsin card sorting test ${ }^{38}$ and a letter fluency task); general intellectual abilities (components of the Wais- $\mathrm{R}^{39}$ ); and premorbid IQ (the national adult reading $\mathrm{scale}^{40}$ ). All patients underwent CT or MRI and HMPAOSPECT, together with the usual battery of screening blood tests to exclude treatable causes of dementia.

All patients have participated in a longitudinal Medical Research Council (UK) funded project investigating aspects of language and memory, and have shown progression of their disease with profiles typical of those reported in the literature.

Three groups of patients were involved in the study: frontal lobe variant FTD $(n=13)$, temporal lobe variant FTD or semantic dementia $(n=20)$, and probable $\operatorname{AD}(n=37)$. All patients in the FTD groups fulfilled the recent consensus criteria for frontotemporal lobar degeneration, ${ }^{41}$ which recognises the subtypes of frontotemporal dementia, semantic dementia, and progressive non-fluent aphasia. We have chosen to use FTD as the broad superordinate term with subsequent subclassification of patients into fv FTD and semantic 
dementia according to the pattern of neuropsychological, behavioural, and neuroimaging abnormalities described below.

All patients with fv FTD presented with an informant based history of progressive change in personality and behaviour, with at least five of the following features: loss of insight, disinhibition, apathy, restlessness, emotional lability, distractibility, reduced empathy, impulsivity, social withdrawal, poor self care, and features of Kluver-Bucy syndrome. In addition to these behavioural changes, most of the patients also showed some impairment in executive functioning, as assessed by a verbal fluency task and the modified Wisconsin card sorting test, ${ }^{38}$ and difficulties in planning tasks such as the Tower of London test, ${ }^{42}$ and subtests from the behavioural assessment of the dysexecutive syndrome. ${ }^{43}$ Patients with significant impairment on tests of semantic memory were excluded from this group. Participants with a history of significant head trauma, alcoholism, movement disorder, or any other condition known to impair frontal lobe function were also excluded in line with our locally developed diagnostic criteria for fv FTD. ${ }^{44-46}$ All patients showed either frontal atrophy on MRI or frontal lobe hypoperfusion on HMPAO-SPECT

Patients with semantic dementia presented with progressive loss of vocabulary affecting expressive and receptive language in the context of fluent speech production. All patients fulfilled the criteria for semantic dementia previously reported: anomia, impairment in single word comprehension, and impoverished semantic knowledge with relative preservation of phonology, syntax, visuospatial abilities, and day to day (episodic) memory..$^{35}$ In all cases, structural brain imaging by MRI showed focal atrophy involving the polar and inferolateral regions of the temporal lobe. In some cases, the atrophy was clearly bilateral, although in others it was markedly asymmetric, if not unilateral.

The diagnosis of probable $\mathrm{AD}$ was made according to the criteria developed by the National Institute of Neurological and Communicative Disorders and Stroke (NINCDS) and the Alzheimer's Disease and Related Disorders Association (ADRDA), which consist of inclusion and exclusion criteria. ${ }^{47}$ All patients presented to the Cambridge Memory Disorders Clinic with a progressive cognitive impairment, predominantly affecting memory. Brain MR scans were either normal or showed a mild degree of medial temporal atrophy.

The patients were grouped according to severity of disease using the mini mental state examination (MMSE): group $1 \mathrm{minimal} /$ very mild (MMSE $>23, \mathrm{n}=18$ ); group 2, mild (MMSE between 18 and 23, $n=27$ ); group 3,

Table 1 Demographic variables of the three patient groups

\begin{tabular}{lllllll}
\hline Group & $n$ & $\begin{array}{l}\text { Age } \\
\text { range }\end{array}$ & $\begin{array}{l}\text { Mean age } \\
(S D)\end{array}$ & $\begin{array}{l}\text { Sex } \\
\text { M/F }\end{array}$ & $\begin{array}{l}\text { MMSE } \\
\text { range }\end{array}$ & $\begin{array}{l}\text { MMSE } \\
\text { mean }\end{array}$ \\
\hline Semantic dementia (tv FTD) & 20 & $49-74$ & $63(6.25)$ & $7 / 13$ & $3-28$ & 16.4 \\
fv FTD & 13 & $54-70$ & $60.2(6.03)$ & $11 / 2$ & $11-30$ & 24.3 \\
AD & 37 & $51-82$ & $71.2(6.95)$ & $19 / 18$ & $8-30$ & 19.2 \\
\hline
\end{tabular}

moderate (MMSE $<18, \mathrm{n}=24)$. These groups correspond to $0.5,1$, and 2 on the clinical dementia rating scale (CDR) ${ }^{48}$ All except three of the patients were living at home, those who were institutionalised had a spouse or relative who still maintained close contact and was therefore able to complete the questionnaire.

The patients with Alzheimer's disease were significantly older than both FTD groups: $\left(F_{(2,5)}=14.5, \quad \mathrm{p}<0.001, \quad \mathrm{AD}>\right.$ semantic dementia $>$ fv FTD). There were also significant differences for MMSE between patients with semantic dementia and those with fv FTD: $\left(F_{(2,67)}=3.67, \mathrm{p}<0.05, \mathrm{fv}\right.$ FTD $>$ semantic dementia). This is not surprising in view of the anomia of the semantic dementia group and the known insensitivity of the MMSE to early frontal dysfunction. ${ }^{39}$

ASSESSMENT OF NEUROPSYCHIATRIC FEATURES A questionnaire was designed following a review of the literature to determine the neuropsychiatric symptoms that are commonly reported in FTD and/or AD. It incorporated questions related to symptoms reported in our previous studies of frontal dementia, ${ }^{44}{ }^{45} \mathrm{com}-$ ponents from the neuropsychiatric inventory, ${ }^{1}$ and specific questions relating to stereotypic behaviour.

After initial piloting, the final version of the questionnaire consisted of 39 questions investigating the following broad domains: depression, elation, irritability, anxiety, aggression, distractibility, executive functioning, risk taking, empathy, apathy, ritualistic/stereotypic behaviour, aberrant motor behaviour, disinhibition, social withdrawal, hallucinations, delusions, changes in food preference, personal care, and sleep patterns (the full questionnaire is available from the authors).

Carers were asked to rate the frequency of a behaviour on a scale of $0-3$. Where a score of zero referred to no change; a score of 1 to occasional occurrence (less than once a week); 2 to a frequent problem (at least once a week); and 3 to a severe behavioural problem. The questionnaire was scored by totalling the carer ratings for all the questions relating to each neuropsychiatric feature; this gave an idea of the prevalence of each of the symptoms.

Test-retest reliability was established by asking 14 of the carers to complete a second questionnaire, 1 week after the first, without reference to it. We found an overall high degree of reliability $\left(r_{\mathrm{s}}=0.86, \mathrm{p}<0.01\right)$ which applied to each of the three patient groups: $\mathrm{AD}\left(r_{\mathrm{s}}=0.89\right.$, $\mathrm{n}=7, \mathrm{p}<0.01)$, semantic dementia $\left(r_{\mathrm{s}}=0.70\right.$, $\mathrm{n}=4, \mathrm{p}<0.01)$, fv FTD $\left(r_{\mathrm{s}}=0.94, \mathrm{n}=3, \mathrm{p}<0.01\right)$.

\section{Results}

The data from the 39 questions were reduced using a principal component factor analysis with varimax factor rotation. This technique is based on the assumption that underlying constructs explain the relation between observed variables.

From an initial scree plot, four principal factors were extracted that best describe the pattern of data collected from these patients (table 
Table 2 Results of factor analysis showing the questions which load on each factor and their eigenvalues

\begin{tabular}{lcccc}
\hline & Factor I & Factor II & Factor III & Factor IV \\
\hline Clockwatching & 0.78 & 0.06 & 0.17 & 0.06 \\
Ritualistic behaviour & 0.75 & 0.14 & 0.15 & -0.01 \\
Use of a catchphrase & 0.65 & 0 & 0.21 & 0.2 \\
Sweet food & 0.61 & 0.08 & -0.03 & -0.03 \\
Appetite increase & 0.6 & 0.21 & 0.21 & -0.01 \\
Counting/numbers & 0.51 & 0.19 & 0.01 & 0.24 \\
Mental rigidity & 0.49 & 0.14 & 0.53 & 0.19 \\
Self-care & 0.25 & 0.64 & -0.04 & 0.32 \\
Poor judgement & 0.2 & 0.64 & -0.04 & 0.32 \\
Lack of planning & 0 & 0.63 & 0.26 & 0.13 \\
Aberrant motor behaviour & 0.21 & 0.6 & 0.24 & -0.27 \\
Distractibility & 0.28 & 0.6 & 0.27 & -0.02 \\
Apathy & 0.18 & 0.56 & -0.02 & 0.48 \\
Mood changes & 0.18 & 0.1 & 0.78 & 0.22 \\
Irritability & 0.11 & 0.23 & 0.76 & 0.15 \\
Aggression & 0 & 0.19 & 0.67 & 0.15 \\
Depression & -0.02 & 0.15 & 0.58 & 0.22 \\
Disinhibition & -0.07 & -0.05 & 0.14 & 0.55 \\
Reduced interest in family & 0.37 & 0.41 & 0.14 & 0.53 \\
Conversational disinhibition & 0.1 & -0.04 & 0.23 & 0.53 \\
Social withdrawal & 0.35 & 0.46 & -0.16 & 0.51 \\
Reduction in conversation & 0.39 & 0.44 & -0.01 & 0.5 \\
Lack of empathy & 0.51 & 0.08 & 0.39 & 0.49 \\
\hline
\end{tabular}

2). The symptoms were considered to be part of the factor if their loading was greater than 0.5.

By far the strongest factor $(E=10.31)$, accounting for $26.4 \%$ of the variance, which we refer to as stereotypic and eating behaviour, consisted of questions related to ritualised behaviour, clockwatching, stereotyped use of catchphrases, preoccupation with counting and numbers, appetite increase, and changes in food preference towards sweet food.

The remaining three factors were weaker. Factor $2(\mathrm{E}=2.97)$ accounting for $7.6 \%$ of the variance, consisted of questions related to poor judgement, lack of planning, aberrant motor behaviour, distractibility and poor self care which we refer to as executive dysfunction and self care.

Factor $3(E=2.49)$ accounting for $6.4 \%$ of the variance, lends itself to the term mood changes, revealing heavy loading of questions related to rapid mood changes, depression, and aggression.

Factor $4(\mathrm{E}=2.18)$ accounting for $5.6 \%$ of the variance, we have called loss of social awareness, consisted of questions about disinhibited behaviour, conversational disinhibition, reduction in conversation, and social withdrawal.

There were a few symptoms which seemed to contribute to more than one factor. Lack of empathy, for instance, was just above the cut off at 0.51 in factor 1 and just below, at 0.496 , in factor 4 . In this case we think that it was more logically in factor 4 along with questions on social withdrawal.

Mental rigidity was just below the cut off for inclusion in factor 1 , at 0.49 , and just above the cut off in factor 3, at 0.53 , but in view of its association with stereotypic and ritualistic behaviour we have included it in factor 1 .

Apathy loaded almost equally into factors 2 and 4. Because mood change and cognitive factors are likely to contribute to apathy we have considered this symptom separately.

INTERGROUP COMPARISONS

Having established the four principal factors, we then investigated differences between groups on these four measures, using two way analysis of variance (ANOVA) according to diagnosis (AD, semantic dementia, fv FTD) and severity of disease (minimal, mild, and moderate). Specific pairwise comparisons were explored using Tukey's HSD post hoc test. Patients who typically showed these behaviours had high, positive scores on the factors, whereas those patients in whom these behaviours were typically absent had low, negative scores.

Factor 1-Stereotypic and eating behaviour Analysis showed a highly significant main effect of diagnosis $\left(F_{(2,67)}=12.6, \mathrm{p}<0.001\right)$. Post hoc analysis showed significant differences between $\mathrm{AD}$ (mean score $=-0.59$ ) and both the other groups, but not between fv FTD (mean=0.40) and semantic dementia (mean score $=0.83$ ). Severity of disease was not significant $\left(F_{(2,67)}\right.$ $<1$ ), and there was no interaction between diagnosis and severity $\left(F_{(4,61)}<1\right)$.

Factor 2-Executive dysfunction and self care By contrast with factor 1, ANOVA disclosed a significant main effect of severity $\left(F_{(2,67)}=4.48\right.$, $\mathrm{p}<0.05)$, with significant differences between minimal (mean score $=-0.31$ ) and moderate (mean score $=0.59$ ), and between mild (mean score $=-0.38$ ) and moderate. However, in this case, there was no significant effect of diagnosis $\left(F_{(2,67)}=1.37, \mathrm{p}=0.26\right)$ and no interaction $\left(F_{(4,61)}<1\right)$.

\section{Factor 3-Mood changes}

For this factor there was no significant effect of diagnosis $\left(F_{(2,67)}<1\right)$ or severity $\left(F_{(2,67)}=1.56\right.$, $\mathrm{p}=0.22)$ and no interaction $\left(F_{(4,61)}<1\right)$.

\section{Factor 4-Loss of social awareness}

As with factor 1 , there was a significant effect of diagnosis $\left(F_{(2,67)}=4.50, \mathrm{p}<0.05\right)$, but in this instance post hoc analysis showed significant differences between fv FTD (mean score $=0.60$ ) and $\mathrm{AD}$ (mean score $=-0.25$ ) only. There was no significant effect of severity $\left(F_{(2,67)}<1\right)$ and no interaction $\left(F_{(4,61)}<1\right)$.

Although the ANOVAs based upon the factor scores establish the pattern of significant differences between groups, these analyses do not consider the frequency of individual symptoms within each group or which symptoms within the cluster are significantly more common in which group. To explore this important clinical issue, we examined the frequency of key symptoms, using the clusters determined above in the three patient groups. It will be recalled that carers used a four point scale. We decided to include scores of 2 or greater as a positive score. Based on this dichotomy, the frequency of the most important symptoms is shown in the figure.

The symptom which most clearly separated the groups was the use of a catchphrase which occurred in $80 \%$ of patients with semantic dementia, $77 \%$ of patients with fv FTD, and only $13 \%$ of patients with AD. This difference was highly significant $\left(\chi_{(2)}^{2}=27.5, \mathrm{p}<0.001\right)$. Mental rigidity was also extremely common, being present in $80 \%$ of the semantic dementia 

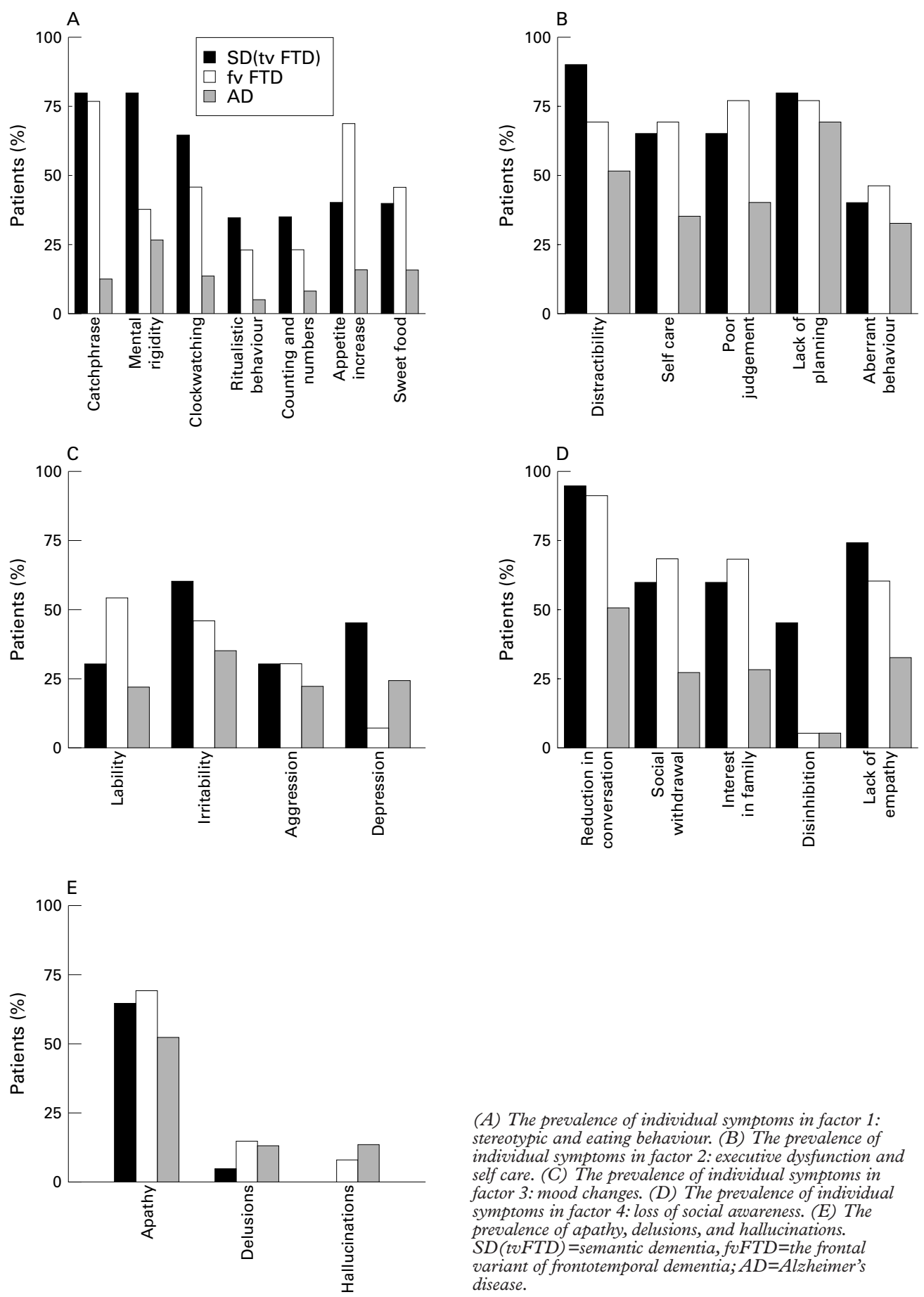

patients, $38 \%$ of $\mathrm{fv}$ FTD, and $27 \%$ of AD $\left(\chi_{(2)}^{2}=13.5, \mathrm{p}<0.01\right)$. Stereotypic/ritualistic behaviour (such as sticking to a very fixed routine or carrying out rituals while doing certain activities) and a preoccupation with counting/ numbers were less common, being present in $35 \%$ of patients with semantic dementia, $23 \%$ with fv FTD, and only $13 \%$ with AD $\left(\chi_{(2)}^{2}=8.40, \mathrm{p}<0.05\right)$

The symptoms which constituted factor 2 ; poor planning/judgement, distractibility, aberrant motor behaviour, and poor self care were present in a surprisingly high proportion of all patient groups (figure B). For instance, distractibility was present in $90 \%$ of semantic

(A) The prevalence of individual symptoms in factor 1: stereotypic and eating behaviour. (B) The prevalence of individual symptoms in factor 2: executive dysfunction and self care. (C) The prevalence of individual symptoms in factor 3: mood changes. (D) The prevalence of individual symptoms in factor 4: loss of social awareness. (E) The prevalence of apathy, delusions, and hallucinations. $S D(t v F T D)=$ semantic dementia, fvFTD=the frontal variant of frontotemporal dementia; $A D=$ Alzheimer's disease.

dementia, $69 \%$ of fv FTD, and $51 \%$ of AD; this difference is significant $\left(\chi_{(2)}^{2}=8.69, \mathrm{p}<0.05\right)$. Decrease in self care also showed a significant difference, occurring in $69 \%$ of fv FTD, $65 \%$ of semantic dementia, and only $35 \%$ of $\mathrm{AD}$ $\left(\chi_{(2)}^{2}=6.99, p<0.05\right)$. The only other significant difference in this factor was poor judgement, which was reported in $77 \%$ of patients with $\mathrm{fv}$ FTD, $65 \%$ of semantic dementia, and $40 \%$ of $\operatorname{AD}\left(\chi_{(2)}^{2}=6.42, \mathrm{p}<0.05\right)$.

Figure $\mathrm{C}$ shows that mood related symptoms, including irritability, were less commonly present and were more or less equally prevalent in all groups, with scores being slightly raised in semantic dementia and fv FTD. The exception 
was depression, which was present in $45 \%$ of patients with semantic dementia, $24 \%$ of patients with $\mathrm{AD}$, and only $7 \%$ of the patients with fv FTD $\left(\chi_{(2)}^{2}=0.11\right)$.

The prevalence of the symptoms in the final cluster, loss of social awareness was also high across all the groups, but several were present to a higher degree in the FTD subgroups (figure D). Reduction in conversation was present in $95 \%$ of patients with semantic dementia, $92 \%$ of $\mathrm{fv} F \mathrm{FT}$, and $51 \%$ of AD; this difference was significant $\left(\chi_{(2)}^{2}=15.6, \mathrm{p}<0.01\right)$. Social withdrawal occurred in $69 \%$ of patients with $\mathrm{fv}$ FTD, $60 \%$ of those with semantic dementia, and only $27 \%$ of those with $\mathrm{AD}\left(\chi_{(2)}^{2}=10.6\right.$, $\mathrm{p}<0.05)$. Similar percentages showed a reduced interest in the family but this difference did not quite reach significance. Disinhibition was fairly prevalent in the fv FTD group, $46 \%$ compared with just $5 \%$ of the semantic dementia group and the AD group. Significant differences were found in both conversational disinhibition $\left(\chi_{(2)}^{2}=17.7, \mathrm{p}<0.001\right)$ and disinhibited behaviour $\left(\chi_{(2)}^{2}=7.65, \mathrm{p}<0.05\right)$. These two questions have been combined on the graph to give an overall measure of disinhibiton.

Delusions were reported in $15 \%$ of patients with fv FTD, $11 \%$ with AD, and only 5\% with semantic dementia (figure E). Hallucinations were even less commonly reported and occurred in $15 \%$ of patients with $\mathrm{AD}, 8 \%$ of patients with fv FTD, and none of the patients with semantic dementia. None of these differences were significant.

Apathy was reported in $73 \%$ of patients with fv FTD, $65 \%$ of semantic dementia, and only $54 \%$ of AD. This difference was not significant.

DISCRIMINANT ANALYSIS

A stepwise discriminant function analysis was performed to determine which symptom clusters differentiated the patient groups with the highest diagnostic accuracy. Factor 1-stereotypic and eating behaviour - was shown to discriminate between the patient groups most reliably, followed by factor 4-loss of social awareness. It is not surprising, following the results of the ANOVAs, that factors 2 and 3-executive dysfunction and self care and mood changes-did not contribute to the discriminant analysis.

Overall, $71.4 \%$ of patients were correctly classified, $55 \%$ of patients with semantic dementia, $86.5 \%$ of patients with $\mathrm{AD}$, and $53.8 \%$ of patients with fv FTD. The patients with fv FTD tended to be misclassified as semantic dementia $(38.5 \%)$, with only a small proportion being misclassified as $\mathrm{AD}(7.7 \%)$. Of the patients with semantic dementia, $20 \%$ were misclassified as $\mathrm{AD}$ and $25 \%$ as fv FTD. Only a small proportion of patients with AD were not correctly classified and they were equally misclassified as semantic dementia $(5.4 \%)$ and fv FTD $(8.1 \%)$.

\section{Discussion}

As predicted, our behavioural questionnaire disclosed striking differences between patients with FTD and $\mathrm{AD}$, although the number of domains in which such differences existed was perhaps less than expected from published literature. Of the four symptom clusters disclosed by factor analysis only two, stereotypic and eating behaviour (factor 1) and loss of social awareness (factor 4), were significantly more common in the FTD group. By far the strongest factor was the former, accounting for $26 \%$ of the overall variance. Of the symptoms within this cluster, the use of a catchphrase, mental rigidity, and alteration in appetite were by far the most discriminating. Within factor 4, reduction in conversation, social withdrawal, loss of interest in family, and disinhibition were the principal symptoms which separated FTD and $\mathrm{AD}$. It is interesting to note that neither mood nor dysexecutive symptoms distinguished the groups. In previous studies many symptoms have been listed as characteristic of FTD. ${ }^{20}{ }^{26}{ }^{45} \mathrm{It}$ is clear, however, from this study that only certain symptoms clearly distinguish FTD from AD.

Rather surprisingly, we found no significant differences between patients with semantic dementia and fv FTD using the factor analysis. Examination of the proportion of patients scoring highly on the individual symptoms showed, however, definite but subtle differences. The patients with semantic dementia scored much more highly on mental rigidity and depression than the patients with fv FTD. Conversely, patients with fv FTD were more disinhibited.

We were particularly interested in the range and frequency of stereotypical behaviour in FTD and included a wider range of questions than previous workers. We found routine, mental rigidity, clockwatching, use of a catchphrase, ritualised behaviour, and a preoccupation with counting and numbers to be increased in both of the FTD groups compared with AD. Only factor 2-executive dysfunction and self care - showed an effect of severity of disease, with the more impaired patients scoring more highly on these questions.

Overall, our results support the findings of Miller $e t a l^{4}$ in their study evaluating the Lund/ Manchester criteria. They found 12 out of the 17 symptoms that they investigated to be significantly higher in patients with FTD than in patients with AD. Eleven of these symptoms were included in our questionnaire and we found the same significant differences except for distractibility and impulsivity, which were equally prevalent in FTD and AD. Miller et al reported that loss of personal awareness, eating, perseverative behaviour, and a reduction in speech most clearly differentiated FTD from $\mathrm{AD}$, three out of four of these symptoms were included in our questionnaire (not loss of personal awareness) and were found to be significantly increased in the FTD group.

Like Levy et $a l^{22}$ we found patients with FTD to show increased levels of disinhibition and lower levels of depression. Apathy, euphoria, and aberrant motor behaviour were, however, equally prevalent in all of our patient groups. Lebert et $a l^{50}$ using the frontotemporal behavioural scale, found increased scores in FTD on all four of their symptom clusters; self 
monitoring, self neglect, self centred behaviour, and mood changes. Our results support these findings with the exception of mood symptoms, which were found to be equally common in both FTD and AD. Depression was only reported in one of our patients with $\mathrm{fv}$ FTD.

Of the symptoms in our study that overlap with those in the frontal behavioural inventory, ${ }^{28}$ only disinhibition was found to be raised in FTD compared with AD. In a pilot study using this instrument, Kertesz et al found higher levels of disinhibition, euphoria, aggression, and perseveration in FTD.

It is clear, therefore, that patients with $\mathrm{fv}$ FTD and semantic dementia are behaviourally very similar and that the main distinction is that the second results in marked semantic deficits. The high prevalence of behavioural changes in semantic dementia may have been overlooked in the past because of the profound linguistic deficits which interfere with patient evaluation. It is also possible that the behavioural symptoms occur at a later stage in semantic dementia.

Turning to the neuroanatomical implications of our findings, it is likely that the behavioural syndrome reflects the involvement of a common network in both variants of FTDnamely, the ventral (orbitobasal) frontal lobe, temporal pole, and amygdala. ${ }^{51}$ The two clusters of symptoms which distinguished FTD from $\mathrm{AD}$, stereotypic behaviour, change in food preference, and loss of social awareness have all been linked to ventral frontal pathology. It seems likely that this region is affected from an early stage in all patients with both variants, either by direct pathological involvement or indirectly via damage to the temporal pole and amygdala, which are heavily interconnected with the ventromedial frontal lobe. The ventral frontal cortex has long been associated with regulating inhibitory control and damage leads to a failure to inhibit inappropriate responses. ${ }^{52}$ Based on these well established findings, Plaisted and Sahakian ${ }^{53}$ proposed that deficits in social cognition in patients with frontal lobe damage are due to an inability to inhibit inappropriate behaviours. They argued that this prevents the selection of more appropriate action plans dictated by long term goals. An alternative explanation comes from Damasio et $a P^{4}$ who proposed that damage to the ventromedial frontal cortices leads to an inability to activate somatic states linked to punishment and reward previously associated with specific social situations. Normally, these are activated in connection with anticipated outcomes of response options. A third theory for the breakdown in social behaviour is the "theory of mind" view, which suggests that these patients have lost the ability to make inferences about the mental states of others. ${ }^{55}$ This view is supported by the finding that these patients do show a loss of empathy for others, and observations that conversation becomes increasingly self centred.

Edwards Lee et $a l^{20}$ reported many of the same behavioural deficits in temporal lobe variant FTD and suggested that either the temporal lobes themselves are important mediators of social behaviour, or the ventromedial frontal cortex and anterior temporal structures work reciprocally. There is strong evidence from both human and monkey studies to support the second view. Medial temporal lobe lesions affecting the limbic system including the amygdala have long been associated with behavioural deficits. ${ }^{56}$ Ablation studies in monkeys have shown that damage to the ventromedial frontal cortex leads to disturbances of emotional and social behaviour. ${ }^{56} 57$

In humans, the ventromedial frontal cortex has also been implicated in decision making, ${ }^{58}$ risk assessment, ${ }^{59}$ and in pathophysiology of obsessive compulsive disorders. ${ }^{27}{ }^{60}$ In keeping with these findings Mummery et $a l^{61}$ suggest that behavioural disturbances in FTD arise from disruption to a network of limbic regions, including the ventromedial frontal cortex, amygdala, and connections to anterior temporal lobe structures.

The differences in behavioural profiles between patients with the two variants of FTD are also of potential interest. Patients with $\mathrm{fv}$ FTD showed higher levels of disinhibition and very low reports of depression, whereas patients with semantic dementia showed greater mental rigidity. The latter group experience a gradual loss of expressive and receptive vocabulary; clinging to a fixed routine and becoming extremely rigid in their thinking may, therefore, be an attempt to maintain some control and understanding over a world that is becoming increasingly incomprehensible.

By contrast with the clear separability of the groups by symptoms related to putative ventromedial frontal pathology, it is interesting that we found an effect of severity only for factor 2 (dysexecutive function and self care). Dysexecutive ability reflects dorsolateral prefrontal functioning and this region is involved relatively late in the course of $\mathrm{AD}$, when the disease has spread from the hippocampal and posterior association cortex. ${ }^{62}$ We hypothesise that in FTD, pathology also spreads upwards to involve this region late in the course of the disease. Decrease in levels of self care could reflect problems with planning and organisation, or might be considered to be a consequence of general apathy.

Changes in mood, except for depression, were equally common in all three patient groups and seemed, therefore, to be of limited diagnostic value. Depressed mood may occur as a psychological response to a diagnosis of a neurodegenerative disease, or it may be caused by changes in the neurochemical systems. The lack of depression found in patients with $\mathrm{fv}$ FTD in this study accords with previous findings and may reflect the almost universal lack of insight found in these patients. ${ }^{25}$

Apathy was commonly reported in all patient groups, with scores being higher in semantic dementia and fv FTD, although the prevalence was rather less than previously reported in both $\mathrm{AD}$ and FTD. ${ }^{22}{ }^{64}$ It is also surprising that delusions and hallucinations were present in such a small minority of patients from all three groups. Many studies have looked at the preva- 
lence of these symptoms in $\mathrm{AD}$ and the results have varied widely. Delusions have been found to affect $10 \%$ to $73 \%,{ }^{65-71}$ and hallucinations $3 \%$ to $49 \% .^{65}{ }^{69-74}$ Our results are more in keeping with those of Cummings ${ }^{75}$ who found delusions to affect $22 \%$ of patients and hallucinations $10 \%$ of patients with $\mathrm{AD}$.

Our study has, of course, some potential shortcomings. Firstly, many of the patients included were quite late in the course of their disease. We attempted to compensate for this by the segregation of cases by disease severity, although the MMSE is not a very satisfactory instrument for this purpose in FTD. ${ }^{39}$ In future work, it would obviously be desirable to include more cases newly presenting to the clinic. Secondly, it might be argued that a study of this type entails a degree of circularity in that the patients with fv FTD were selected, partly, on the basis of prominent behavioural symptoms (in addition to radiological and neuropsychological features). It should be emphasised, however, that the features found to discriminate FTD from AD were a specific subset of a wider range used to select cases. Moreover, the patients with semantic dementia were selected on the basis of cognitive and neurological features, yet were found to have a high prevalence of neuropsychiatric symptoms. Finally, any study of this type must be regarded as preliminary, our findings require confirmation in a new prospectively assessed cohort.

Overall, our questionnaire correctly classified $71.4 \%$ of the patients involved in this study and only $13 \%$ of patients with AD were incorrectly classified. It is hoped that it will prove to be a useful tool for discriminating between the dementias both in a clinical and research setting. This has become increasingly important as therapies specifically targeted at $\mathrm{AD}$ have emerged and are sought for FTD. Changes in personality and behaviour are often the earliest symptoms of dementia of the frontal type and are usually the most distressing aspect of the disease for the carers. At presentation, these patients may perform flawlessly on conventional cognitive assessments, it is, therefore, imperative to find ways of accurately quantifying neuropsychiatric features if we are to be able to diagnose patients early in the course of the disease.

We are indebted to the continuing support of the patients and carers included in this study. We are also very grateful to Peter Watson for his statistical advice. This work was supported as part of a Medical Research Council (UK) programme grant to JRH. MALR is supported by a National Institutes of Health (USA) grant.

1 Cummings JL, Mega M, Gray K, et al. The neuropsychiatric inventory: comprehensive assessment of psychopathology in dementia. Neurology 1994;44:2308-14

2 Brun A, Englund B, Gustafson L, et al. Clinical and neuropathological criteria for frontotemporal dementia. $\mathcal{F}$ Neurol Neurosurg Psychiatry 1994;57:416-18.

3 Gregory CA, Hodges JR. Frontotemporal dementia: use of consensus criteria and prevalence of psychiatric features. Neuropsychiatry Neuropsychology and Behavioural Neurology 1996;9:145-53.

4 Miller BL, Ikonte C, Ponton M, et al. A study of the LundManchester research criteria for frontotemporal dementia: clinical and single photon emission CT correlations. Neurology 1997;48:937-42.

5 Snowden JS, Neary D, Mann DMA. Fronto-temporal lobar degeneration: fronto-temporal dementia, progressive aphasia, degeneration: fronto-temporal dementia, progressive aphasia,
semantic dementia. New York: Churchill Livingstone, 1996.

6 Hodges JR. Pick's disease: It's relationship to semantic dementia, progressive aphasia and frontotemporal demen- tia. In: O'Brien J, Ames D, Burns A, ed. Dementia. Arnold: London, 2000, 741-58.

7 Pick A. Uber die Beziehungen der senilen Hirnatrophie zur Aphasie. Prager Medicinische Wochenschrift 1892;17:165-7. 8 Pick A. Senile Hirnatrophie als Gundlage von Hernderscheinunger, Wiener Klinische Wochenschrift. Monatschrift für Psychratrie und Neurologie 1901;14:403-4.

9 Pick A. Uber einen weiteren symptomenkomplex im Rahmen der Dementia senilis, bedingt durch umschriebene starkere Hirnatrophie (gemische Apraxie). Monatschrift für Psychratrie und Neurologie 1906;19:97-108.

10 Brun A. Provisional criteria for a diagnosis of frontal lobe dementia. Archives of Gerontology and Geriatrics 1987;6: 193-208.

11 Neary D, Snowden JS, Northen B, et al. Dementia of frontal lobe type. $尹$ Neurol Neurosurg Psychiatry 1988;51:353-61.

12 Miller BL, Cummings JL, Villanueva-Meyer J, et al. Frontal obe degeneration: clinical, neuropsychological, an SPECT character

13 Jagust WL, Reed BR, Seab JP, et al. Clinical-physiologic correlates of Alzheimer's disease and frontal lobe dementia. Am f Physiol Imaging 1989;4:89-96.

14 Hodges JR, Garrard P, Patterson K. Semantic dementia. In: Kertesz A, Munoz DG, eds. Pick's disease and Pick complex. New York: Wiley-Liss, 1998:83-104

15 Garrard P, Hodges JR. Semantic dementia: implications for the neural basis of language and meaning. Aphasiology 1999;13:609-23.

16 Rahman S, Sahakian BJ, Hodges JR, et al. Specific cognitive deficits in early frontal variant frontotemporal dementia. Brain 1999;122:1469-93.

17 Mesulam MM. Slowly progressive aphasia without generalised dementia. Ann Neurol 1982;11:592-8.

18 Hodges JR, Patterson K. Non-fluent progressive aphasia and semantic dementia: a comparative neuropsychological and semantic dementia: a comparative neurops

19 Mummery CJ, Patterson K, Wise RJS, et al. Disrupted temporal lobe connections in semantic dementia. Brain 999;122:61-73

20 Edwards Lee T, Miller B, Cummings J, et al. The temporal lobe variant of frontotemporal dementia. Neurology 1996; 46:2023.

21 Spillantini MG, Bird TD, Ghetti B. Frontotemporal dementia and parkinsonism linked to chromosome 17: a new group of tauopathies. Brain Path 1998;8:387-402.

22 Levy ML, Miller BL, Cummings JL, et al. Alzheimer disease and frontotemporal dementias: behavioral distinctions. Arch Neurol 1996;53:687-90.

23 Pachana NA, Boone KB, Miller BL, et al. Comparison of neuropsychological functioning in Alzheimer's disease and frontotemporal dementia. F Int Neuropsychol Soc 1996;2: 505-10.

24 Hodges JR, Patterson K, Garrard P, et al. The differentiation of semantic dementia and frontal lobe dementia (temporal and frontal variants of fronto-temporal dementia) from early Alzheimer's disease: a comparative neuropsychological study. Neuropsychology 1999;13:31-40.

25 Gregory CA, McKenna PJM, Hodges JR. Dementia of frontal type and simple schizophrenia: two sides of the same coin? Neurocase 1998;4:1-6.

26 Gustafson L. Frontal lobe degeneration of non-Alzheimer type. II. Clinical picture and differential diagnosis. Archives of Gerontology and Geriatrics 1987;6:209-33.

27 Ames D, Cummings JL, Wirshing WC, et al. Repetitive and compulsive behavior in frontal lobe degenrations. $f$ Neuropsychiatry Clin Neurosci 1994;6:100-13.

28 Kertesz A, Munoz DG. Pick's disease and Pick complex. New York: Wiley-Liss, 1998.

29 Snowden JS, Goulding PJ, Neary D. Semantic dementia: a form of circumscribed cerebral atrophy. Behav Neurol $1989 ; 2: 167-82$.

30 Hodges JR, Patterson K, Oxbury S, et al. Semantic dementia: progressive fluent aphasia with temporal lobe atrophy. Brain 1992;115:1783-806.

31 Warrington EK. Recognition memory test. Windsor: NFER Nelson, 1984

32 Wechsler DA. Wechsler memory scale: revised. San Antonio: Psychological Corporation, 1987.

33 Rey A. L'examen psychologique dans les cas d'encephalopathie traumatique. Arch Psychol (Frankf) 1941;28:286-340.

34 Bozeat S, Lambon Ralph MA, Patterson K, et al. Non-verbal semantic impairment in semantic dementia. Neuropsychologia (in press).

35 Hodges JR, Graham N, Patterson K. Charting the progression in semantic dementia: implications for the organisation of semantic memory. Memory 1995;3:463-95.

36 Howard D, Patterson K. Pyramids and palm trees: a test of semantic access from pictures and words. Bury St Edmunds, Suffolk: Thames Valley Test Company, 1992

37 Warrington EK, James M. The visual object and space perception battery. Bury St Edmunds: Thames Valley Test Company, 1991.

38 Nelson HE. A modified card sorting test sensitive to frontal lobe deficits. Cortex 1976;12:313-24.

39 Wechsler DA. Wechsler adult intelligence scale: revised. Test manual. New York: Psychological Corporation, 1981.

40 Nelson HE. National adult reading scale (NART):test manual. 2nd ed. Windsor, UK: NFER-Nelson, 1982.

41 Neary D, Snowden JS, Gustafson L, et al. Frontotemporal lobar degeneration: a consensus on clinical diagnostic criteria. Neurology 1998;51:1546-54.

42 Shallice T. Specific impairments in planning. Philos Trans $R$ Soc Lond 1982;298:199-209. 
43 Wilson BA, Alderman N, Burgess PW, et al. Behavioural assessment of the dysexecutive syndrome. (BADS). Bury $\mathrm{St}$ assessment of the dysexecutive syndrome. (BADS).

44 Gregory CA, Hodges JR. Dementia of frontal type and the focal lobar atrophies. International Reviews of Psychiatry 1993;5:397-406.

45 Gregory CA, Hodges JR. Clinical features of frontal lobe dementia in comparison with Alzheimer's disease. $\mathcal{F}$ Neural Trans 1996;47:103-23.

46 Gregory CA, Orrell M, Sahakian B, et al. Can frontotemporal dementia and Alzheimer's disease be differentiated using a brief battery of tests? International fournal of Geriatric Psychiatry 1997;12:375-83.

47 McKhann G, Drachman D, Folstein M, et al. Clinical diagnosis of Alzheimer's disease: report of the NINCDSADRDA work group under the auspices of department of health and human services task force on Alzheimer's disease. Neurology 1984;34:939-44.

48 Hughes CP, Berg L, Danziger WL, et al. A new clinical scale for the staging of dementia. B f Psychiatry 1982;140:566for the

49 Gregory CA, Hodges JR. The differentiation of frontotemporal dementia and Alzheimer's disease. Alzheimer's Disease Society Newsletter 1998;3:67

50 Lebert F, Pasquier F, Souliez L, et al. Frontotemporal behavioural scale. Alzheimer Dis Assoc Disord 1998;12:3359.

51 Mummery CJ, Patterson K, Hodges JR, et al. Functional neuroanatomy of the semantic system: divisible by what? Fournal of Cognitive Neuroscience 1998;10:766-77.

52 Luria AR. The neuropsychology of memory. Washington: Winston, 1976.

53 Plaisted KC, Sahakian BJ. Dementia of frontal lobe type: living in the here and now. Aging and Mental Health 1997; $1: 293-5$.

54 Damasio AR, Tranel D, Damasio H. Individuals with sociopathic behaviour caused by frontal damage fail to respond autonomically to social stimuli. Behav Brain Res 1990;41: autonom

55 Stone VE, Baron-Cohen S, Knight RT. Frontal lobe contributions to theory of mind. fournal of Cognitive Neuroscience butions to theory
1998;10:640-56.

56 Butter CM, Snyder DR, McDonald JA. Effects of orbitofrontal lesions on aversive and aggressive behaviour in rhesus monkeys. Fournal of Comparative and Physiological Psychology 1970;72:132-44.

57 Myers RE, Swett CS, Miller M. Loss of social group affinity following prefrontal lesions in free-ranging macaques. Brain Res 1973;64:257-69.

58 Bechara A, Damasio H, Tranel D, et al. Dissociation of working memory from decision making within the human prefrontal cortex. F Neurosci 1998;18:428-37.
59 Rogers RD, Sahakian BJ, Hodges JR, et al. Dissociating executive mechanisms of task control following frontal lobe lamage and Parkinson's disease. Brain 1998;121:815-42.

60 Zald DH, Kim SW. Anatomy and function of the orbital frontal cortex 2. Function and relevance to obsessive compulsive disorder. F Neuropsychol Clin Neurosci 1996;8:?

61 Mummery CJ, Patterson K, Price CJ, et al. A voxel based morphometry study of semantic dementia: the relationship between temporal lobe atrophy and semantic dementia. Ann Neurol 2000;47:36-45.

62 Braak H, Braak E. Neuropathological staging of Alzheimerrelated changes. Acta Neuropathologica 1991;82:239-59.

63 Brun A, Englund E. Regional pattern of degeneration in Alzheimer's disease: neuronal loss and histopathological grading. Histopathology 1981;5:549-64.

64 Mega MS, Cummings JL, Fiorello T, et al. The spectrum of behavioural changes in Alzheimer's disease. Neurology 1996;46:130-5.

65 Birkett DP. The psychiatric differentiation of senility and arteriosclerosis. Br F Psychiatry 1972;120:321-5.

66 Cummings JL, Miller B, Hill MA, et al. Neuropsychiatric aspects of multi-infarct dementia and dementia of the Alzheimer type. Arch Neurol 1987;44:389-93.

67 Kumar A, Koss E, Metzler D. Behavioural symptomatology in dementia of the Alzheimer's type. Alzheimer Dis Assoc Disord 1988;2:363-5.

68 Merriam AE, Aronson MK, Gaston P, et al. The psychiatric symptoms of Alzheimer's disease. I Am Geriatr Soc 1988;36:7-12.

69 Wragg RE, Jeste DV. Overview of depression and psychosis in Alzheimer's disease. Am f Psychiatry 1989;146:577-87.

70 Copper JK, Mungas D, Weiler PG. Relation of cognitive status and abnormal behaviours in Alzheimer's disease. $7 \mathrm{Am}$ Geriatr Soc 1990;38:867-70.

71 Cohen D, Eisdorfer C, Gorelick P. Psychopathology associated with Alzheimer's disease and related disorders. $f$ Gerontol 1993;48:255-60.

72 Berrios GE, Brook P. Delusions and psychopathology of the elderly with dementia. Acta Psychiatr Scand 1985;72:296301.

73 Gilley DW, Whalen ME, Wilson RS, Bennett DA Hallucinations and associated factors in Alzheimer's disease. $\mathcal{F}$ Neuropsychiatry Clin Neurosci 1991;3:371-6.

74 Sinha D, Zemlan FP, Nelson S. A new scale for assessing behavioural agitation in dementia. Psychiatry Res 1992;41: $73-88$.

75 Cummings JL. The neuropsychiatric inventory: assessing psycholpoathology in dementia patients. Neurology 1997; 48:S10-6. 